J. Math. Kyoto Univ. (JMKYAZ)

11-1 (1971) 45-49

\title{
Commutative rings which are locally Noetherian
}

\author{
By \\ J. T. Arnold and J. W. Brewer \\ (Communicated by Professor Nagata, May 4, 1970)
}

It is well known that if $R$ is a Noetherian commutative ring, then $R_{M}$, the localization of $R$ at each maximal ideal $M$, is also Noetherian. Any almost Dedekind domain ${ }^{1)}$ which is not Dedekind provides an example for which the converse fails [1, App. 1, Ex. 1]. We shall say that a ring $R$ is locally Noetherian provided $R_{M}$ is Noetherian for each maximal ideal $M$ of $R$. The goal of this paper is to characterize those locally Noetherian rings which are also Noetherian.

Throughout this paper $R$ will denote a commutative ring with identity. Our notation and terminology are essentially that of [1] with the following exception: if $S$ is a multiplicatively closed subset of $R$ and if $A$ is an ideal of $R$, then we shall denote by $A R_{S}$ the extension of $A$ to $R_{S}$.

\section{The Characterization Theorem}

Let $A$ be any ideal of $R$ and let $S$ be the set of elements of $R$ which are not zero divisors modulo $A$. Then $S$ is multiplicatively closed and $A \cap S=\varnothing$. Any prime ideal containing $A$ which is maximal with respect to missing $S$ is called a maximal prime divisor of $A$. A prime ideal $P$ of $R$ is called a prime divisor of $A$ if there is a multi-

1) If $D$ is an integral domain with identity, then $D$ is said to be an almost Dedekind domain if $D_{M}$ is a Noetherian valuation ring for each maximal ideal $M$ of $D$ [1, p. 408]. 
plicatively closed subset $S_{1}$ of $R$ such that $A \cap S_{1}=\varnothing$ and $P R_{S_{1}}$ is a maximal prime divisor of $A R_{S_{1}}[2, \mathrm{p} .19]$. In the sequel the term prime divisor will be used only in the above sense. It is clear that any prime ideal of $R$ which is minimal with respect to the property of containing $A$ is a prime divisor of $A$ and such prime ideals are called minimal prime divisors of $A$.

In order to prove our main theorem, we require some preliminary results.

Lemma 1.1. Let $R$ be a ring which satisfies the ascending chain condition (a.c.c.) for prime ideals and suppose that each finitely generated ideal of $R$ has only finitely many minimal prime divisors. If $P$ is any proper prime ideal of $R$, then $P$ is the unique minimal prime divisor for some finitely generated ideal $A$ of $R$.

Proof. Let $P$ be a proper prime ideal of $R$ and let $x \in P-\{0\}$. By assumption, $(x)$ has only finitely many minimal prime divisors-say $P_{1}, \ldots, P_{n}$, and since $P \supseteq(x), P \supseteq P_{i}$ for some $i$. Suppose that $P \neq P_{i}$ for $1 \leq i \leq r$, where either $r=n$ or $r=n-1$, and let $x_{i} \in P-P_{i}$. If $A=\left(x, x_{1}, \ldots, x_{r}\right)$, then $A$ has only finitely many minimal prime divisors-say $P_{1}^{\prime}, \ldots, P_{m}^{\prime}$. Since $P_{i}^{\prime} \supseteq A \supseteq(x), P_{i}^{\prime}$ contains a minimal prime divisor of $(x)$. If $P_{i}^{\prime} \supseteq P$, then $P$ is a minimal prime divisor of $A$ and $P_{i}^{\prime}=P$, since $P_{i}^{\prime} \supseteq P \supseteq A$. If $P_{i}^{\prime} \supseteq P_{j}$ for some $j, 1 \leq j \leq r$, then the containment is proper for we have $x_{j} \in P_{i}^{\prime}-P_{j}$. But $R$ satisfies $a$. c. c. for prime ideals so it follows that, after finitely many repetitions of the above procedure, we may obtain a finitely generated ideal $A_{1}$ having unique minimal prime divisor $P$.

Corollary 1.2. Let $R$ be a ring which satisfies the a.c.c. for prime ideals. Then $R$ is Noetherian if and only if each finitely generated ideal $A$ of $R$ has only finitely many minimal prime divisors and $\sqrt{A}$ is finitely generated. 
Proof. By Lemma 1.1, if $P$ is a proper prime ideal of $R$, then $P$ is the unique minimal prime divisor for some finitely generated ideal $A$ of $R$. Thus, $P=\sqrt{A}$ and $P$ is finitely generated. It follows that $R$ is Noetherian $[1$, p. 26]. The converse is clear.

Lemma 1.3. If $R$ is a locally Noetherian ring, then $R$ satisfies a.c.c. on prime ideals.

Proof. Let $P_{1} \subseteq P_{2} \subseteq \ldots$ be a chain of prime ideals of $R$ and set $P=U_{i=1}^{\infty} P_{i}$. Then $P$ is a prime ideal of $R$ and $R_{p}$ is Noetherian [2, 6.5 and 6.11]. Since $P_{1} R_{p} \subseteq P_{2} R_{p} \subseteq \ldots \subseteq P R_{p}$ is a chain of prime ideals of $R_{p}$, there is an integer $n$ such that $P R_{p}=P_{n+i} R_{p}$ for each nonnegative integer $i$. Hence, $P=P_{n+i}$ for each nonnegative integer $i$ and the lemma follows.

We are now able to prove our main theorem.

Theorem 1.4. The following conditions are equivalent in a locally Noetherian ring $R$.

(1) Each finitely generated ideal of $R$ may be expressed as a finite intersection of primary ideals of $R$.

(2) Each finitely generated ideal of $R$ has only finitely many prime divisors.

(3) Each finitely generated ideal $A$ of $R$ has only finitely many minimal prime divisors and $\sqrt{A}$ is finitely generated.

(4) $R$ is Noetherian.

Proof. It is clear that $(4) \Rightarrow(1) \Rightarrow(2)$. That (3) and (4) are equivalent is an immediate consequence of Corollary 1.2 and Lemma 1.3. We now show that $(2) \Rightarrow(4)$. Thus, let $P$ be a proper prime ideal of $R$. By Lemma 1.1 and Lemma 1.3, $P$ is the unique minimal prime divisor of some finitely generated ideal $A$ of $R$. If $p_{1}, \ldots, p_{r} \in R$ are such that $P R_{p}=\left(p_{1}, \ldots, p_{r}\right) R_{p}$, then $P$ is the unique minimal prime divisor of $A_{1}=A+\left(p_{1}, \ldots, p_{r}\right)$ and $A_{1} R_{p}=P R_{p}$. Suppose that $P, P_{1}, \ldots, P_{n}$ 
are the prime divisors of $A_{1}$ and let $y_{i_{1}}, \ldots, y_{i m_{i}} \in R$ be such that $P R_{p_{i}}=\left(y_{i 1}, \ldots, y_{i m_{i}}\right) R_{p_{i}}$. For $1 \leq i \leq n, P_{i}$ is not a prime divisor of $A_{2}=A_{1}+\sum_{i=1}^{n}\left(y_{i 1}, \ldots, y_{i m_{i}}\right)$, since $P_{i} R_{p_{i}}$ is not a prime divisor of $A_{2} R_{p_{i}}$ $=P R_{p_{i}}$. Suppose that $Q$ is any proper prime ideal of $R$ which contains $A_{2}$ and suppose that $Q \not P_{i}$ for any $i$. But $Q \supseteq A_{2} \supseteq A_{1}$, so $Q \supseteq P$ and it follows that $P R_{Q}$ is the unique prime divisor of $A_{1} R_{Q}$. Thus, $A_{1} R_{Q}$ is $P R_{Q}$-primary [2, p. 20] and, consequently, if $\left(A_{1} R_{Q}\right)^{e c}$ is the extension and contraction of $A_{1} R_{Q}$ with respect to $\left(R_{Q}\right)_{P R_{Q}}$, then $A_{1} R_{Q}$ $=\left(A_{1} R_{Q}\right)^{e c}=\left[A_{1}\left(R_{Q}\right)_{P R_{Q}}\right]^{c}=\left(A_{1} R_{p}\right)^{c}=\left(P R_{Q}\right)^{c}=P R_{Q}[2$, p. 17]. But $A_{1} R_{Q} \subseteq A_{2} R_{Q} \subseteq P R_{Q}$ so we have $A_{2} R_{Q}=P R_{Q}$. Hence, $Q R_{Q}$ is not a prime divisor of $A_{2} R_{Q}$ and it follows that $Q$ is not a prime divisor of $A_{2}$. Therefore, if $P, P_{1}^{\prime}, \ldots, P_{m}^{\prime}$ are the prime divisors of $A_{2}$, then for $1 \leq i \leq m, P_{i}^{\prime} \supset P_{j}$ for some $j, 1 \leq j \leq n$. Since $R$ satisfies a.c.c. for prime ideals, a finite number of repetitions of the above procedure yields a finitely generated ideal $A_{3}$ such that $A_{3} R_{p}=P R_{p}$ and $P$ is the unique prime divisor of $A_{3}$. Then $A_{3}$ is $P$-primary $[2$, p. 20] so $A_{3} R_{p}=P R_{p}$ implies that $A_{3}=P$. Thus, $P$ is finitely generated and the theorem follows.

In general a ring can satisfy any of the conditions (1), (2) and (3) of Theorem 1.4 without being Noetherian. In fact, if $X=\left\{X_{i}\right\}_{i=1}^{\infty}$ is a countable collection of indeterminates over the field $K$, then $K[X]$ is a non-Noetherian ring in which each of these conditions is satisfied. To see this, let $A=\left(f_{1}, \ldots, f_{r}\right)$ be a finitely generated ideal of $K[X]$. For some integer $n$ we have $f_{1}, \ldots, f_{r} \in D_{n}$, where $D_{n}=K\left[X_{1}, \ldots, X_{n}\right]$. Since $D_{n}$ is Noetherian, $A^{\prime}=\left(f_{1}, \ldots, f_{r}\right) D_{n}$ has a finite irredundant primary representation in $D_{n}$-say $A^{\prime}=Q_{1} \cap \ldots \cap Q_{m}$, and $\sqrt{A^{\prime}}$ is finitely generated. Hence, $A=A^{\prime}[X]$ has the finite irredundant primary representation $A=Q_{1}[X] \cap \ldots Q_{m}[X]$ and $\sqrt{A}=\left(\sqrt{ }^{\prime}\right)[X]$ is finitely gen erated.

No two of the conditions (1), (2) and (3) are necessarily equivalent in an arbitrary ring $R$. Any rank one nondiscrete valuation ring satisfies property (1) but not (3). For an example of a ring in which (2) holds but neither (1) nor (3) holds, consider any rank two valuation 
ring $V$. Property (1) holds only if principal ideals of $V$ are primary, but if $P$ is the minimal prime ideal of $V$ and $x \in P-\{0\},(x)$ is not primary [1, p. 173]. If $M$ is the maximal ideal of $V$ and $y \in M-P$, then either $\sqrt{(y)}=M$ is not finitely generated or $\sqrt{(x)}=P$ is not finitely generated $[1$, p. 73$]$.

Department of Mathematics

Virginia Polyteghnic Institute Blacksburg, Virginia 24061 (USA)

\section{References}

1. Gilmer, R. W., Jr.: Multiplicative Ideal Theory, Queen's Papers on Pure and Applied Mathematics-No. 12, Kingston, Ontario, 1968.

2. Nagata, M.: Local Rings: New York: Interscience 1962. 